\title{
ANALGESIA PÓS-OPERATÓRIA
}

\section{POSTOPERATIVE ANALGESIA}

\section{Betina Sílvia Beozzo Bassanezi ${ }^{1}$; Antonio Gonçalves de Oliveira Filho - TCBC-SP²}

\begin{abstract}
RESUMO: Justificativas e Objetivos: A dor sempre foi uma das maiores preocupações do homem, entretanto, apesar dos progressos da ciência, ainda existem várias barreiras ao seu adequado tratamento, incluindo a falta de conhecimento por parte da equipe médica, sobre o mecanismo das diversas drogas e técnicas empregadas. O objetivo deste trabalho é abordar as principais drogas e técnicas empregadas no controle da dor pós-operatória, visando estimular o interesse sobre o assunto bem como aumentar a eficácia do tratamento dado aos pacientes. Conteúdo: Está ressaltada neste artigo, a importância da adequada analgesia pós-operatória, considerando as principais drogas e técnicas utilizadas no controle da dor, seus mecanismos de ação, posologias, vias de administração e efeitos colaterais, bem como a importância da integração de toda a equipe envolvida nos cuidados do paciente para o sucesso do tratamento. O tratamento inadequado da dor no pós-operatório não se justifica, pois há um arsenal considerável de drogas e técnicas analgésicas. O que se faz necessário, portanto, é que toda equipe, anestesistas, cirurgiões, e enfermeiros tenham conhecimento e estejam integrados na utilização deste arsenal (Rev. Col. Bras. Cir. 2006; 33(2): 116-122).
\end{abstract}

Descritores: Analgesia; Dor pós-operatória/prevenção e controle; Dor pós-operatória/terapia: Analgésicos; Cirurgia.

\section{INTRODUÇÃO}

A dor é um sintoma comum nas doenças cirúrgicas, tanto no momento do diagnóstico como no pós-operatório.

O conceito de que a dor pós-operatória é normal e esperada, associado à falta de conhecimento da fisiologia da dor e da farmacologia dos analgésicos bem como a falta de treinamento da equipe de enfermagem para avaliação do quadro álgico, faz com que a atenção da equipe esteja voltada às complicações pós-operatórias mais comuns (fístulas, infecções, sangramentos, etc.) do que ao sintoma que mais incomoda o paciente: a dor. O resultado disso é que grande parte dos pacientes cirúrgicos experimenta dor intensa no pós-operatório. Em um estudo realizado no Hospital das Clínicas de São Paulo verificou-se que somente $20 \%$ dos pacientes apresentavam controle adequado da dor nas primeiras 24 horas de pós-operatório e $50 \%$ deles apresentavam intensa dor nas 48 horas iniciais ${ }^{1}$. Em estudo realizado nos Estados Unidos com 500 pacientes operados, $77 \%$ deles apresentavam dor pósoperatória, sendo que em $80 \%$ dos casos a dor era classificada como moderada a intensa ${ }^{2}$.

A dor não é apenas um incômodo, mas envolve complexas reações fisiológicas, com manifestações autonômicas e psicológicas que levam à imunossupressão, à diminuição da perfusão tissular, ao aumento do consumo de oxigênio, do trabalho cardíaco, ao espasmo muscular, à alteração da mecânica respiratória e à liberação dos hormônios do "stress", culminando no aumento do catabolismo e alteração do balanço nitrogenado. Além disso, a não mobilização do paciente, decorrente da dor, aumenta o risco de ocorrência de pneumonia e trombose venosa no período pós-operatório, havendo relação direta destes eventos com o aumento da morbi-mortalidade do paciente cirúrgico ${ }^{3-4}$.

Nos hospitais dos EUA, atualmente, estão se formando os serviços de dor aguda (APS- acute pain service), que têm por objetivo a aplicação de métodos eficientes para o controle da dor pós-operatória, além da educação da equipe de médicos e enfermeiros ${ }^{1,2}$.

A incidência e intensidade da dor dependem de características individuais, do tipo de operação, e da qualidade do tratamento instituído. Com relação às características individuais, além das influências culturais, sociológicas e de personalidade, tem se demonstrado uma correlação inversamente proporcional entre as necessidades de analgésicos e as concentrações de endorfinas e norepinefrina no líquor ${ }^{4}$. Com relação ao tipo de operação, a intensidade da dor no pósoperatório está mais na dependência do local operado do que da gravidade do procedimento, obedecendo a seguinte ordem decrescente: cirurgia torácica , abdominal superior, abdominal inferior, ortopédica e as cirurgias periféricas ${ }^{4}$.

Portanto, a qualidade do tratamento da dor instituído no pós-operatório é a única variável onde a equipe pode e tem a obrigação de interferir visando uma recuperação mais tranqüila do paciente.

Esta revisão tem por objetivo alertar as equipes cirúrgicas da necessidade do tratamento adequado da dor pósoperatória e também fornecer bases teóricas sobre as drogas mais usadas na atualidade para o seu controle.

1. Médica Anestesiologista do Centro Infantil Boldrini.

2. Professor Doutor da Disciplina de Cirurgia Pediátrica da FCM UNICAMP; Cirurgião Oncológico Pediátrico do Centro Infantil Boldrini. 


\section{PREVENÇÃO DA DOR PÓS-OPERATÓRIA}

Os estudos neurofisiológicos da dor mostram que a repetição de estímulos nociceptivos modifica a percepção dolorosa e facilita sua transmissão. Isto se explica através da ampliação do campo de receptores cutâneos e diminuição das influências inibitórias da dor, principalmente pela ação dos receptores N-Metil-D-aspartato. Portanto, teoricamente, a inibição prévia das vias nociceptivas através de um bloqueio perineural com anestésicos locais ou administração de opióides ou antiinflamatórios, impediriam a sua hiperexcitabilidade e diminuiriam a dor pós-operatória. Estes são os princípios do que se chama analgesia preempitiva, onde a escolha da técnica anestésica e a utilização de analgésicos previamente ao ato operatório influenciariam diretamente a qualidade do controle da dor pós-operatória ${ }^{5}$. No entanto, ainda existem controvérsias sobre a eficácia da analgesia preempitiva devido à dificuldade de comprovação de que a qualidade analgésica de determinada técnica seja melhor quando esta é administrada antes do estímulo cirúrgico ${ }^{4}$.

Apesar da controvérsia existente, os bloqueios peridurais mostram-se efetivos na diminuição do catabolismo nitrogenado, resultado da liberação dos hormônios do stress (ex.: glucagon, catecolaminas e cortisol), das repercussões pulmonares decorrentes do procedimento cirúrgico e conseqüentemente da morbi-mortalidade. Por esses fatores a analgesia peridural associada à anestesia geral tem-se tornado cada vez mais comum na prática anestésica ${ }^{3,4,6,7}$.

\section{TRATAMENTO}

O tratamento da dor pós-operatória deve ser feito de forma regular e não de demanda, atendendo as necessidades individuais de cada paciente, devendo haver familiaridade com a técnica e as drogas escolhidas, especialmente por parte da equipe médica e de enfermagem, que cuida do paciente.

\section{Antiinflamatórios não esteróides (AINE)}

Nessa categoria de fármacos estão incluídas as drogas que agem através da inibição da ciclooxigenase (COX), bloqueando a conversão do ácido araquidônico em prostanóides, conhecidos como prostaglandinas e prostaciclinas e tromboxanos, envolvidos no processo inflamatório e na sensibilização dolorosa central e periférica. Aparentemente os AINE também possuem uma ação inibitória na transmissão nociceptiva, sinérgica aos agonistas alfa2 e opiáceos. A ação dos AINE é dose /resposta limitada (efeito teto), ou seja, a sua administração em doses superiores às recomendadas não proporciona analgesia suplementar, aumentando a incidência de efeitos colaterais.

Atualmente se conhecem dois tipos de COX. ACOX1, presente na maioria dos tecidos está relacionada à função renal (balanço de água e sódio), à agregação plaquetária e à proteção da mucosa gástrica. A COX-2 normalmente presente no sistema nervoso central e no aparelho urogenital, é induzida durante o processo inflamatório se expressando nos macrófagos e outras células dos tecidos inflamados. Portanto a maior parte dos efeitos colaterais dos AINE, está relacionada a utilização de AINE não seletivos e de ação na COX-1, principalmente irritação gastrointestinal e alteração da hemostasia. Sua administração deve ser feita com extrema cautela em pacientes com patologias gastroduodenais, renais, ou com alteração da hemostasia ${ }^{3,4,8,9}$. Os AINE, COX-2 seletivos, têm sido relacionados ao aumento da incidência de fenômenos tromboembólicos como infarto agudo do miocárdio e acidente vascular cerebral, o que levou a retirada de alguns destes fármacos do mercado.

Os AINE, no controle da dor pós-operatória, têm sido utilizados por via endovenosa devido ao caráter doloroso da via intramuscular e à dificuldade do emprego da via oral imediatamente após o ato operatório, quando o paciente ainda se encontra sonolento ou em jejum.

No Brasil os AINE que podem ser utilizados via endovenosa se restringem ao cetoprofeno $(100$ a $300 \mathrm{mg} / \mathrm{dia}$ diluídos em 100ml de solução isotônica), ao tenoxicam (20 a $40 \mathrm{mg} / \mathrm{dia}$ )e ao cetarolaco (10-30mg/dia).

\section{Dipirona e Acetaminofeno}

Apesar da dipirona e do acetaminofeno serem as medicações analgésicas e antipiréticas mais populares, os seus mecanismos de ação permanecem controversos. Alguns estudos sugerem que a inibição da COX-2 possa explicar os efeitos farmacológicos da dipirona e do acetaminofeno ${ }^{10}$ Levy et $a l .{ }^{11}$ demonstraram a redução de tromboxano no líquor humano após a utilização de dipirona, o que poderia explicar seu efeito analgésico e antipirético. Outros autores descreveram um terceiro tipo de $\mathrm{COX}$ (COX-3), presente principalmente no córtex cerebral, que é inibida seletivamente por drogas analgésicas e antipiréticas, como a dipirona e o acetaminofeno. Assim a inibição da COX-3 pode representar o mecanismo primário central da ação analgésica destas drogas ${ }^{12}$. Nos Estados Unidos da América e na Inglaterra a utilização da dipirona foi restrita pela sua associação com agranulocitose. Mas estudos na Europa, Ásia e América Latina têm demonstrado que o risco de agranulocitose, anemia aplástica, anafilaxia e sérias complicações gastrointestinais com o uso da dipirona é baixo, em torno de 25/milhão, semelhante ao do acetaminofeno (20/milhão) e muito menor que o do ácido acetilsalicílico (185/ milhão ${ }^{10}$. A dose recomendada de dipirona é de $15 \mathrm{mg} / \mathrm{kg}$ a cada 6 horas $^{6}$ e do acetaminofeno é de $12 \mathrm{mg} / \mathrm{kg}$ a cada 4 a 6 horas $^{13}$.

\section{Opiáceos}

A ação analgésica deste grupo de fármacos se deve à ocupação de receptores mu, kappa e delta, reforçando a ação fisiológica das endorfinas e a das vias inibitórias noradrenérgicas e serotoninérgicas. Inibem, ainda, a liberação de neuromediadores da dor, como a substância P, e hiperpolarizam os neurônios aferentes do corno posterior da medula. A ação periférica dos opiáceos se explica através da ligação com receptores das terminações nervosas livres, ativos na presença de reação inflamatória ${ }^{3,4}$. 
Na tentativa de dissociar a ação analgésica dos efeitos de depressão respiratória dos opiáceos, surgiram os agonistas parciais, dos quais se destacam a buprenorfina e os agonistas-antagonistas (agonistas kappa e antagonistas $\mathrm{mu}$ ), como a pentazocina e a nalbufina. Na prática clínica a diminuição do potencial de depressão respiratória destas drogas se relaciona também à diminuição da potência analgésica ${ }^{4}$.

O tramadol é classificado como opióide por sua ação agonista sobre os receptores mu, mas apenas 30\% de sua ação analgésica é revertida pela naloxona. Também atua sobre a inibição da recaptação de norepinefrina e serotonina e facilita a liberação pré-sináptica de serotonina. Sua potência analgésica é de 5 a 10 vezes menor que a da morfina, mas alguns estudos têm demonstrado uma eficácia semelhante, comparável a da morfina por via epidural, no controle da dor em cirurgias de grande porte. Na prática clínica a utilização do tramadol não tem sido associada à depressão respiratória, e apresenta um baixo potencial para o desenvolvimento de tolerância, dependência ou abuso ${ }^{14,15}$.

Como efeitos colaterais, os analgésicos opióides podem provocar sedação, náusea, vômitos, prurido, retenção urinária e constipação intestinal numa relação dose-dependente. Altas doses de opióides podem levar à depressão respiratória, apnéia, colapso circulatório e coma seguido de morte 4 .

Os opiáceos são efetivos no controle da dor e podem ser administrados praticamente por todas as vias e diferentes doses (Tabela 1) ${ }^{16-24}$. A administração de opióides lipossolúveis via epidural, como o fentanil e o sufentanil, é controversa, uma vez que na prática clínica as doses necessárias por via epidural para analgesia se assemelham às doses endovenosas, provavelmente a ação via epidural se deva primeiramente a absorção sistêmica e subseqüente distribuição cerebral, e não por ação direta na medula espinhal ${ }^{17-21}$. Portanto, a utilização de uma via de administração mais invasiva e de maior custo

Tabela 1- Analgesia pós-operatória: opióides, doses, vias de administração.

\begin{tabular}{|c|c|c|c|}
\hline Analgésicos Opióides & Posologia & Vias de Administração & Ref \\
\hline Tramadol & $\begin{array}{l}50-100 \mathrm{mg}-2 \text { a } 3 \text { x / dia } \\
\text { dose máxima } 400 \mathrm{mg} \\
10 \text { a } 20 \mathrm{mg} / \mathrm{h} \\
20-100 \mathrm{mg} / \text { dia }\end{array}$ & $\begin{array}{l}\text { VO, IV ou IM } \\
\text { IV (infusão contínua) } \\
\text { Epidural }\end{array}$ & $\begin{array}{l}15 \\
23\end{array}$ \\
\hline Meperidina & $\begin{array}{l}2,5 \text { a } 3,5 \mathrm{mg} / \mathrm{kg} \text { a cada } 3 \mathrm{a} 4 \mathrm{~h} \\
1,5 \mathrm{a} 2,0 \mathrm{mg} / \mathrm{kg} \text { a cada } 3 \mathrm{a} 4 \mathrm{~h} \\
1,5 \mathrm{a} 2,0 \mathrm{mg} / \mathrm{kg} \text { a cada } 3 \mathrm{a} 4 \mathrm{~h} \\
0,3 \mathrm{a} 0,6 \mathrm{mg} / \mathrm{kg} / \mathrm{h} \\
30 \mathrm{a} 100 \mathrm{mg} \text { a cada } 4 \mathrm{a} 6 \mathrm{~h} \\
14 \mathrm{a} 20 \mathrm{mg} / \mathrm{h} \\
0,2-1,0 \mathrm{mg}\end{array}$ & $\begin{array}{l}\text { VO } \\
\text { SC } \\
\text { IV (bolus) } \\
\text { IV (em infusão contínua) } \\
\text { Epidural (bolus) } \\
\text { Epidural (infusão contínua) } \\
\text { Intratecal }\end{array}$ & $\begin{array}{l}4 \\
4 \\
4 \\
4 \\
17,23 \\
22 \\
4\end{array}$ \\
\hline Morfina & $\begin{array}{l}0,5 \mathrm{a} 1,0 \mathrm{mg} / \mathrm{kg} \text { a cada } 3 \mathrm{a} 4 \mathrm{~h} \\
0,15 \mathrm{mg} / \mathrm{kg} \text { a cada } 3 \mathrm{a} 4 \mathrm{~h} \\
0,15 \mathrm{mg} / \mathrm{kg} \text { a cada } 3 \mathrm{a} 4 \mathrm{~h} \\
0,03 \mathrm{a} 0,1 \mathrm{mg} / \mathrm{kg} / \mathrm{h} \\
0,1 \mathrm{mg} \mathrm{a} 1 \mathrm{mg} \text { dose única } \\
30 \text { a } 50 \mu \mathrm{g} / \mathrm{kg} \text { a cada } 8 \text { a } 24 \mathrm{~h} \\
0,4 \text { a } 0,6 \mathrm{mg} / \mathrm{h} \\
1 \text { a } 10 \mathrm{mg}\end{array}$ & $\begin{array}{l}\text { VO } \\
\text { SC } \\
\text { IV (bolus) } \\
\text { IV (infusão contínua) } \\
\text { Intratecal } \\
\text { Epidural (bolus) } \\
\text { Epidural (infusão contínua) } \\
\text { Intra-articular (diluir em 20ml de SF 0,9\%) }\end{array}$ & $\begin{array}{l}4 \\
4 \\
4 \\
18 \\
23 \\
19 \\
17,23 \\
24\end{array}$ \\
\hline Fentanil & $\begin{array}{l}\text { Dose inicial } 0,8 \mu \mathrm{g} \text { a } 1,6 \mu \mathrm{g} / \mathrm{kg} \\
0,3 \mathrm{mg} \text { a } 1,6 \mu \mathrm{g} / \mathrm{kg} / \mathrm{h} \\
50 \text { a } 200 \mathrm{mg} \text { a cada } 2 \text { a } 5 \mathrm{~h} \\
5-20 \mu \mathrm{g} \\
75 \mathrm{mg} \text { a } 150 \mathrm{mg} \text { a cada } 48 \text { a } 72 \mathrm{~h}\end{array}$ & $\begin{array}{l}\text { IV (infusão contínua) } \\
\text { Epidural (infusão contínua) } \\
\text { Epidural (bolus) } \\
\text { Intratecal } \\
\text { Transdérmico }\end{array}$ & $\begin{array}{l}4 \\
17,23 \\
17 \\
23 \\
16\end{array}$ \\
\hline Sufentanil & $\begin{array}{l}2-8 \mu \mathrm{g} \\
15 \text { a } 50 \mu \mathrm{g} \text { a cada } 4 \text { a } 6 \mathrm{~h} \\
0,15 \text { a } 0,3 \mu \mathrm{g} / \mathrm{kg} / \mathrm{h}\end{array}$ & $\begin{array}{l}\text { Intratecal } \\
\text { Epidural (bolus) } \\
\text { Epidural (infusão contínua) }\end{array}$ & $\begin{array}{l}23 \\
17,23 \\
17\end{array}$ \\
\hline Nalbufina & 5 a $10 \mathrm{mg}$ a cada $4 \mathrm{~h}$ & IV, $\mathrm{SC}$ & 23 \\
\hline Buprenorfina & $0,4 \mathrm{mg}$ a cada 6 a $8 \mathrm{~h}$ & SL & 16,23 \\
\hline
\end{tabular}


Tabela 2 - Analgesia pós-operatória: Anestésicos locais, doses, vias de administração.

\begin{tabular}{llll}
\hline Anestésicos Locais & Doses & Vias deAdministração & Ref \\
\hline Levobupivacaina & $6 \mathrm{ml} / \mathrm{h} \mathrm{a} 0,25 \%$ & Epidural (infusão contínua) & 27,28 \\
Ropivacaína & $20 \mathrm{ml} \mathrm{a} \mathrm{0,75 \%}$ & Intra-articular( joelho) & 29 \\
& $5 \mathrm{ml} / \mathrm{h} \mathrm{a} 0,2 \%$ & Interescalênico (infusão contínua) & 26 \\
& $10 \mathrm{a} 20 \mathrm{ml} \mathrm{a} 0,2 \%$ & Epidural (bolus) & 25 \\
& $6-14 \mathrm{ml} / \mathrm{h} \mathrm{a} \mathrm{0,2 \%}$ & Epidural (infusão contínua) & 25 \\
& $4 \mathrm{a} 8 \mathrm{ml} / \mathrm{h}$ & Epidural torácica (infusão contínua) & 25 \\
& & & 3,18 \\
Bupivacaína & $5-10 \mathrm{ml} \mathrm{a} 0,5 \%$ a cada $4 \mathrm{~h}$ & Epidural (bolus) & 30 \\
& $3-10 \mathrm{ml} / \mathrm{h}$ a $0,25 \%$ & Epidural (infusão contínua) & 29 \\
& $5-15 \mathrm{ml} / \mathrm{h} \mathrm{a} 0,125 \%$ & Intra-articular (joelho) & 26 \\
& $20 \mathrm{a} 30 \mathrm{ml} \mathrm{a} 0,5 \%$ & Interescalênico (infusão contínua) & 31 \\
& $5 \mathrm{ml} / \mathrm{h} \mathrm{a} 0,15 \%$ & Inter-pleural & 18,32 \\
& $15 \mathrm{ml} \mathrm{a} 0,5 \%$ cada $8 \mathrm{~h}$ & Inter-pleural (infusão contínua) & 33 \\
\hline
\end{tabular}

para o controle da dor não se justificaria. Nos Estados Unidos, não é permitido o emprego do fentanil por via transdérmica no tratamento da dor aguda.

A meperidina possui lipossolubilidade intermediária, tendo sua ação analgésica mediada predominantemente pela medula espinhal quando utilizada a via epidural em pequenas doses, e teoricamente com menores efeitos colaterais quando comparada à morfina. A meperidina é metabolizada no fígado, formando a normeperidina, com importantes propriedades excitatórias no sistema nervoso centra (SNC)l e uma meia-vida longa, em torno de 15 horas, podendo chegar a 35 horas em pacientes com insuficiência renal. $\mathrm{O}$ aumento das concentrações plasmáticas de normepiridina limita o uso da meperidina por tempo prolongado ou em altas doses ${ }^{22,23}$.

\section{Anestésicos locais}

Os anestésicos locais atuam sobre os canais de sódio das terminações nervosas, bloqueando a transmissão do estímulo nociceptivo. Sua utilização pode ser feita por diferentes vias e doses (subaracnóidea, peridural, perineural, intra-articular, interpleural, intercostal, inter-escalênica, infiltração da ferida cirurgica) - Tabela 2, com o objetivo de proporcionar somente analgesia, mantendo-se a sensibilidade táctil e a motricidade ${ }^{24-33}$. $\mathrm{O}$ anestésico local deve ser empregado em baixas concentrações dando-se preferência a bupivacaína em aplicações intermitentes ou infusão contínua. A ropivacaína tem sido descrita como tendo menor ação sobre as fibras motoras (dissociação sensitivo-motora) e menor toxicidade cardiocirculatória em relação à bupivacaína racêmica, destacando-se como anestésico local para o controle da dor pósoperatória. Da mesma maneira, a levobupivacaína também possui uma menor cardiotoxicidade em relação à sua forma racêmica, com eficácia clínica similar ${ }^{25-27,34,36}$.

As restrições quanto ao uso exclusivo dos anestésicos locais para analgesia pós-operatória dizem respeito ao fenômeno de taquifilaxia que exige um aumento progressivo na dose utilizada, e aos efeitos indesejáveis de hipotensão arterial e de bloqueio motor quando utilizado em infusão contínua ${ }^{26,27,34-36}$. A associação de opiáceos, clonidina, ou cetamina via espinhal ou endovenosa diminui estes fenômenos e proporciona uma melhor analgesia ${ }^{17,37-39}$.

\section{Agonistas Alfa-2}

Os receptores $a_{2}$ estão localizados nas terminações pré-sinápticas das fibras simpáticas e sua estimulação inibe a produção e liberação da norepinefrina. No SNC os receptores $\mathrm{a}_{2}$ encontram-se no tronco cerebral e a ativação de seus núcleos resulta em sedação e anestesia. Também no tronco cerebral, a ação dos agonistas a ativa uma via inibitória descendente da medula espinhal, diminuindo o tônus simpático. $\mathrm{Na}$ medula espinhal estes receptores estão localizados no corno posterior e sua ativação inibe a transmissão da informação dolorosa, resultando em analgesia ${ }^{40-42}$.

A clonidina, agonista $\mathrm{a}_{2}$-adrenérgico, tem sido usado extensivamente em anestesiologia, inclusive na analgesia pósoperatória. Sua atividade analgésica foi inicialmente descrita em pacientes oncológicos que se tornaram tolerantes a morfina e tiveram sua dor atenuada após o emprego de clonidina por via peridural ${ }^{43}$. A clonidina também pode ser administrada por via endovenosa, no entanto para a analgesia pós-operatória a utilização por via epidural ou epineural se mostrou mais efetiva. A dose recomendada, por via epidural, é de 1 a $8 \mathrm{mg} / \mathrm{kg}$ em bolus ou 0,08 a $2 \mathrm{mg} / \mathrm{kg} / \mathrm{h}$, ou ainda de 10 a $50 \mathrm{mg} / \mathrm{h}^{17,41,44}$. Seu uso isolado não produz analgesia adequada no pós-operatório e está associado à sedação intensa e hipotensão arterial. Por outro lado sua associação com opióides e anestésicos locais resulta numa diminuição do consumo de analgésicos. Syngelyn et $a l .{ }^{44}$, sugere que a utilização da clonidina com anestésicos locais, para bloqueio de nervos periféricos, proporciona maior duração de analgesia no pós-operatório, 
sem os efeitos colaterais indesejáveis da associação com opióides.

\section{Cetamina}

A cetamina em pequenas doses possui efeitos analgésicos, através de sua ação antagonista nos receptores $\mathrm{N}$ metil D-aspartato localizados no SNC, incluindo a medula espinhal. Previne a sensibilização do SNC em resposta à estimulação nociceptiva periférica. Isto explicaria sua prolongada ação analgésica, mesmo após uma dose única ou infusões contínuas por períodos curtos ${ }^{4,42}$.

A cetamina atua ainda sobre o sistema cardiovascular através do aumento de catecolaminas circulantes, o que resulta em elevação da freqüência cardíaca e da pressão arterial. Apresenta ação direta sobre a musculatura lisa dos vasos, provocando vaso dilatação, sendo seu efeito sobre o miocárdio ainda discutível. Esta droga deprime pouco a ventilação através da diminuição da resposta respiratória ao $\mathrm{CO}_{2}$, mas normalmente observa-se a manutenção ou o aumento do volume minuto, da atividade da musculatura respiratória intercostal, e normalidade da capacidade residual funcional ${ }^{45}$.

A cetamina tem sido indicada em associação com anestésicos locais e opióides por via epidural ou com opióides por via endovenosa, potencializando a ação analgésica destas medicações. A utilização de cetamina intraoperatória chega a reduzir, em alguns casos, até $50 \%$ da necessidade de morfina no pós-operatório ${ }^{17,38,42}$. Entretanto alguns estudos que avaliaram o emprego da cetamina na analgesia pós-operatória em cirurgias com extensas incisões e manipulações viscerais não demonstraram efetividade no controle da dor ${ }^{46}$.

As doses recomendadas de cetamina são de $0,15 \mathrm{mg} /$ $\mathrm{kg}$ por via endovenosa em injeção única com o objetivo de prevenção de hiperalgesia, ou 1 a $2 \mathrm{mg} / \mathrm{kg} / \mathrm{min}$ por via endovenosa em infusão contínua, ou ainda $0,2 \mathrm{mg}-1,0 \mathrm{mg} / \mathrm{kg}$ por via epidural ${ }^{37,42}$.

\section{Neostigmine}

A neostigmina é um anticolinesterásico, portanto aumenta a concentração de acetilcolina nas sinapses nervosas, que por sua vez age em interneurônios colinérgicos e gabaérgicos do corno posterior da medula com efeito antinociceptivo, atuando sobre a liberação de norepinefrina, glicina e ácido gama-aminobutírico ${ }^{42}$. Alguns estudos têm demonstrado sua ação analgésica quando administrada via epidural em doses de 50 a $60 \mathrm{ìg}$ ou de 5 a $10 \mathrm{ìg} / \mathrm{kg}$. Como efeito colateral mais evidente se observa a hipotensão $0^{47-49}$.

\section{PCA (Patient Controlled-Analgesia)}

PCA é uma modalidade de controle de dor onde existe uma administração de analgésicos por demanda imediata do paciente em quantidade abundante, independente da via de administração e da droga utilizada. Isto diminui o tempo entre o aparecimento do sintoma e o alivio da dor. Habitual- mente empregam-se as vias venosas e epidural, no entanto outras vias, como a subcutânea, têm se mostrado eficazes estando associadas a menor incidência de hipoxemia ${ }^{50}$. Embora qualquer analgésico possa ser administrado por esta técnica, os opióides são os agentes mais empregados.

A infusão contínua de analgésicos facilita o controle e evita flutuações das concentrações plasmáticas com uma analgesia mais eficiente respeitando as diferenças individuais na necessidade de consumo de analgésicos.

Atualmente, as modalidades de PCA mais utilizadas são aquelas com infusão em velocidade constante ou variável e administração sob demanda. Nos casos de velocidade variável existe um microprocessador que monitora a demanda e ajusta a velocidade de infusão. Independente do tipo de PCA existem variáveis básicas para sua utilização: dose inicial (quantidade de medicação necessária para se obter analgesia), intervalo mínimo entre as solicitações do paciente, velocidade de infusão basal e limites.

A utilização de PCA implica em pessoal competente e treinado, pois se faz necessário uma adequada vigilância da qualidade analgésica e efeitos colaterais, principalmente sedação e depressão respiratória. Apesar do seu custo adicional, a PCA tem se tornado bastante popular devido ao grau de satisfação dos pacientes com a utilização desta modalida$\mathrm{de}^{4,51}$.

\section{CONCLUSÃO}

O controle da dor pós-operatória é o primeiro passo para a diminuição da morbi-mortalidade dos pacientes cirúrgicos, pois permite a realização de fisioterapia e deambulação precoces e diminui o "stress" físico e psicológico destes pacientes.

A grande quantidade de artigos com técnicas e medicações analgésicas diversas reforça a idéia de que não existe uma maneira única de se lidar com o tratamento da dor pós-operatória. O que se sugere é a utilização da associação de duas ou mais drogas ou de técnicas analgésicas de classes diferentes, em doses menores, o que constitui a analgesia multimodal ou balanceada que tem como objetivo melhorar a sua qualidade, com menor incidência de efeitos adversos ${ }^{52}$ ${ }^{53}$. A literatura tem nos dado fortes indícios sobre a segurança e efetividade de três técnicas analgésicas: PCA com analgésicos sistêmicos; a analgesia epidural (ou intratecal);e as técnicas de analgesia regional, como o bloqueio intercostal e a infiltração de plexos nervosos e da incisão cirúrgica. Mas somente o envolvimento da equipe, o conhecimento, o treino e a familiaridade com as drogas e com as técnicas empregadas podem garantir a melhoria da qualidade dos cuidados pós-operatórios. É necessário que se estabeleçam protocolos de analgesia pós-operatória de acordo com as características de cada serviço, onde a resposta à terapia analgésica seja regularmente avaliada e documentada já que as condições dos pacientes são dinâmicas e a necessidade de analgésicos pode variar freqüentemente. A adequada avaliação da efetividade analgésica e de seus efeitos colaterais são requisitos indispensáveis para o sucesso no controle da dor pós-operatória. 


\begin{abstract}
Background and Objectives: Pain has been one of the men's biggest worries. Despite of scientific progress there still exist many barriers in an adequate treatment of pain including the lack of knowledge of many drugs and pain management techniques. The objective of this study is to discuss the main drugs and analgesics process in an effort to stimulate our colleague interest about the subject and thus increasing treatment efficiency of our patients. Contents: It is emphasized in this study the importance of an adequate postoperative analgesia discussing the main drugs and techniques used in pain management, their mechanism of action, dose, administration route and side effects of each drug. It is also pointed out the great importance of teamwork with all of the people envolved in patient's care. Conclusions: Nowadays, it is unadmissible that the pain control in postoperative period be done with less than excellent results due to a large choice of drugs and analgesic techniques. Is necessary an adequate interaction of all team members, anesthesiologists, surgeons, and nurses involved in patient's care together with the knowledge of different drugs and techniques for pain management.
\end{abstract}

Key words: Analgesia; Pain, postoperative/prevention \& control; Pain, postoperative/therapy; Analgesics; Surgery.

\section{REFERÊNCIAS}

1. Gozzani JL. Analgesia pós-operatória. In: Manica J. Anestesiologia: princípios e técnicas. $2^{\mathrm{a}}$ ed. Porto Alegre: Artmed; 1997. p. 763-9.

2. Ashburn MA. Future challenges in anesthesia-based acute postoperative pain management. ASA Refresher Courses Anesthesiol. 1999; 27.

3. Lubenow TR, McCarthy RJ. Management of acute postoperative pain. In: Barash PG, Cullen BF, Stoelting RK. Clinical anesthesia. $2^{\text {nd }}$ ed. Philadelphia: Lippincott; 1992. p. 1547-77.

4. Bonnet F, Baubillier F. Analgésie postopératoire. Encycl Méd Chir Anesthésie-Réanimation. 1997; tome 1, 36-550-A-10.

5. Conceição MJ. Analgesia preempitia. In: Manica J. Anestesiologia princípios e técnias. $2^{\text {nd }}$ ed. Porto Alegre: Artmed; 1997. p. 7759.

6. Engquist A, Fog-Moller F, Christiansen C, Thode J, VesterAndersen T, Madsen SN. Influence of epidural analgesia on the catecholamine and cyclic AMP responses to surgery. Acta Anaesthesiol Scand. 1980; 24(1):17-21.

7. Engquist A, Brandt MR, Fernandes A, Kehlet H. The blocking effect of epidural analgesia on the adrenocortical and hyperglycemic responses to surgery. Acta Anaesthesiol Scand. 1977; 21(4):330-5.

8. Desjardins PJ, Grossman EH, Kuss ME, Talwalker S, Dhadda S, Baum D, Hubbard RC. The injectable cyclooxygenase-2-specific inhibitor parecoxib sodium has analgesic efficacy when administered preoperatively. Anesth Analg. 2001; 93(3):721-7.

9. Teixeira MJ. Analgésicos antiinflamatórios não-esteroidais. In: Teixeira MJ. Dor contexto interdisciplinar. $1^{\mathrm{a}}$ ed. [s.1.]: Maio; 2003. p. 571-94.

10. Campos C, de Gregório R, Garcia-Nieto R, Gago F, Ortiz P, Alemany S. Regulation of cyclooxygenase activity by metamizol. Eur J Pharmacol. 1999; 378(3):339-47.

11. Levy M, Brune K, Zylber-Katz E, Cohen O, Caraco Y, Geisslinger G. Cerebrospinal fluid prostaglandins after systemic dipyrone intake. Clin Pharmacol Ther. 1998; 64(1):117-22.

12. Chandrasekharan NV, Dai H, Roos KL, Evanson NK, Tomsik J, Elton TS, Simmons DL. COX-3, a cyclooxigenase-1 variant inhibited by acetaminophen and other analgesic/antipyretic drugs: cloning, structure, and expression. Proc Natl Acad Sci USA, 2002; 99(21):13926-31.

13. Wong A, Sibbald A, Ferrero F,Plager M, Dantolaya ME, Escobar AM, et al. Antipyretic effects of dipyrone versus ibuprofen versus acetaminophen in children: results of multinational, randomized, modified double-blind study. Clin Pediatr. 2001; 40(6):313-24.
14. Eggers KA, Power I. Tramadol. Br J Anaesth. 1995; 74(3):247-49.

15. Bloch MB, Dyer RA, Heijke SA, James MF. Tramadol infusion for postthoracotomy pain relief: a placebo-controlled comparison with epidural morphine. Anesth Analg. 2002; 94(3):523-8.

16. Sandler NA. Novas técnicas de administração de opióide para o controle da dor aguda. Clin Anestesiol Am Norte. 1992; 2:267-82.

17. Badner NH. Agentes administrados por via epidural para analgesia pós-operatória. Clin Anestesiol Am Norte. 1992; 2:317-33.

18. Kavanagh BP, Katz J, Sandler AN. Pain control after thoracic surgery. A review of current techniques. Anesthesiology. 1994; 81(3):737-59.

19. Bernards CM. Epidural and intrathecal opioids: wich drugs should we choose and how should they be used. ASA Refresher Courses Anesthesiol. 1999; 27:13-30.

20. Glass PS, Estok P, Ginsberg B, Goldberg JS, Sladen RN. Use of patient-controlled analgesia to compare the efficacy of epidural to intravenous fentanyl administration. Anesth Analg. 1992; 74(3):345-51.

21. Miguel R, Barlow I, Morrel M, Scharf J, Sanusi D, Fu E. A prospective, randomized, double-blind comparison of epidural and intravenous sufentanil infusions. Anesthesiology. 1994; 81(2):346-52; discussion 25A-26A

22. Ngan Kee WD. Epidural pethidine: pharmacology and clinical experience. Anaesth Intensive Care. 1998; 26(3):247-55.

23. Teixeira MJ. Analgésicos opióides. In: Teixeira MJ. Dor: contexto interdisciplinar. $1^{\text {a }}$ ed. [s.1.]: Maio; 2003. p. 595-612.

24. Drosos GI, Vlachonikolis IG, Papoutsidakis AN, Gavalas NS, Anthopoulos G. Intra-articular morphine and postoperative analgesia after knee arthroscopy. Knee. 2002; 9(4): 335-40.

25. McClellan KJ, Faulds D. Ropivacaine: an update of its use in regional anaesthesia. Drugs. 2000; 60(5):1065-93.

26. Borgeat A, Kalberer F, Jacob H, Ruetsch YA, Gerber C. Patientcontrolled interscalene analgesia with ropivacaine $0.2 \%$ versus bupivacaine $0.15 \%$ after major open shoulder surgery: the effects on hand motor function. Anesth Analg. 2001; 92(1):218-23.

27. McClelland AM, Beckett N, Milligan KR. Post operative analgesia following total hip replacement surgery: A comparison of levobupivacaine and ropivacaine via epidural infusion [abstract]. Anesth Anal. 2002; 94:(2 Suppl):S344.

28. Demedde M, Stadler M, Bardiau F, Boogaerts JG. Continuous epidural infusion of large concentration/small volume versus small concentration/large volume of levobupivacaine for postoperative analgesia. Anesth Analg. 2003; 96(3):796-801.

29. Convery PN, Milligan KR, Quinn P, Sjovall J, Gustafsson U. Efficacy and uptake of ropivacaine and bupivacaine after single intra-articular injection in the knee joint. Br J Anaesth. 2001; 87(4):570-6. 
30. Pouzeratte Y, Delay JM, Brunat G, Boccara G, Vergne C, Jaber $\mathrm{S}$, et al. Patient-controlled epidural analgesia after abdominal surgery: ropivacaine versus bupivacaine. Anesth Analg. 2001; 93(6):1587-92.

31. Reber A, Scheidegger D. An alternative technique for interpleural analgesia. Anesthesiology. 1998; 88(2):553-4.

32. Dryden CM, McMenemin I, Duthie DJ. Efficacy of continuous intercostal bupivacaine for pain relief after thoracotomy. Br J Anaesth. 1993; 70(5): 508-10.

33. McIlvaine WB, Knox RF, Fennessey PV, Goldstein M. Continuous infusion of bupivacaine via intrapleural catheter for analgesia after thoracotomy in children. Anesthesiology.1988; 69(2):261-4.

34. Panos L, Panos AL. Bloqueios local e regional da dor aguda. Clin Anestesiol Am Norte. 1992; 2:335-52.

35. Senard M, Joris J. [Use of ropivacaine for postoperative epidural analgesia]. Ann Fr Anesthesie Reanim. 2002; 21(9):713-24.

36. Murdoch JAC, Dickson UK, Wilson PA, Berman JS, Gad-Elrab RR, Scott NB. The efficacy and safety of three concentrations of levobupivacaine administered as a continuous epidural infusion in patients undergoing orthopedic surgery. Anesth Analg. 2002; 94(2):438-44.

37. De Negri P, Ivani G Visconti C, De Vivo P, Lonnqvist PA. The dose-response relationship for clonidine added to a postoperative continuous epidural infusion of ropivacaine in children. Anesth Analg. 2001; 93(1):71-76.

38. Menigaux C, Guignard B, Fletcher D, Sessler DI, Dupont X, Chauvin M. Intraoperative small-dose ketamine enhances analgesia after outpatient knee arthroscopy. Anesth Analg. 2001; 93(3):606-12.

39. She S, Mok MS, Dong Y, et al. Evaluation of continuous epidural ropivacaine infusion and patient controlled epidural analgesia with morphine-ropivacaine after combined spinal-epidural anesthesia [abstract]. Anesth Analg 2002; 94(2Suppl):S233.

40. Shulman MS. Novos medicamentos para a analgesia sistêmica. Clín Anestesiol Am Norte. 1992; 2:295-316.

41. Eisenach JC. á2-Adrenergic agonists in anesthesia practice. ASA Refresher Courses Anesthesiol. 1999; 27.

42. Lauretti GR. Cetamina, clonidina, neostigmina. In: Teixeira MJ. Dor: contexto interdisciplinar. $1^{\mathrm{a}}$ ed. [s.1.]. Maio; 2003. p. 661-4.

43. Tamsen A, Gordh T. Epidural clonidine produces analgesia. Lancet. 1984; 28(8396):231-2.
44. Singelyn FJ, Dangoisse M, Bartholomée S, Gouverneur JM. Adding clonidine to mepivacaine prolongs the duration of anesthesia and analgesia after axillary brachial plexus block. Reg Anesth. 1992; 17(3):148-50.

45. Fletcher D. Kétamine. Encycl Méd Chir Anesthésie-Réanimation. Tome 1; 36-305-B-30.

46. Reeves M, Lindholm DE, Myles PS. Fletcher H, Hunt JO. Adding ketamine to morphine for patient-controlled analgesia after major abdominal surgery: a double-blinded, randomized controlled trial. Anesth Analg. 2001; 93(1):116-20.

47. Omais M, Lauretti GR, Paccola CA. Epidural morphine and neostigmine for postoperative analgesia after orthopedic surgery. Anesth Analg. 2002; 95(6):1698-701.

48. Nakayama M, Ichinose H, Nakabayashi K, Satoh O, Yamamoto $\mathrm{S}$, Namiki A. Analgesic effect of epidural neostigmine after abdominal hysterectomy. J Clin Anesth. 2001; 13(2):86-9.

49. Kirdemir P, Ozkocak I, Demir T, Gogus N. Comparison of postoperative analgesic effects of preemptively used epidural ketamine and neostigmine. J Clin Anesth. 2000; 12(7):543-8.

50. Doyle E, Morton NS, McNicol LR. Comparison of patientcontrolled analgesia in children by i.v and s.c routes of administration. Br J Anaesth. 1994; 72(5):533-6.

51. Ferrante FM. Analgesia controlada pelo paciente. Clin Anestesiol Am Norte.1992; 2:283-94.

52. Kehlet H, Dahl JB. The value of "multimodal" or "balanced analgesia" postoperative pain treatment. Anesth Analg. 1993; 77(5):1048-56.

53. Practice guidelines for acute pain management in the perioperative setting. A report by the American Society of Anesthesiologists Task Force on Pain Management, Acute Pain Section. Anesthesiology. 1995; 82(4):1071-81.

Como citar este artigo:

Bassanezi BSB, Oliveira Filho AG. Analgesia pós-operatória. Rev Col Bras Cir. [periódico na Internet] 2006 Mar-Abr; 33(2). Disponível em URL: http://www.scielo.br/rcbc

Endereço para correspondência:

Dra. Betina Sílvia Beozzo Bassanezi

R: Prof. Celso Ferraz de Camargo, 217

Cidade Universitária - 13083-200 - Campinas - SP

E-mail: betina@boldrini.org.br 